Efeito da temperatura no desenvolvimento de Euplectrus ronnai (Brèthes) (Hymenoptera, Eulophidae) parasitando lagartas de Pseudaletia sequax Franclemont (Lepidoptera, Noctuidae) e impacto do parasitismo no consumo alimentar do hospedeiro

Effect of temperature on the development of Euplectrus ronnai (Brèthes) (Hymenoptera, Eulophidae) parasitizing Pseudaletia sequax Franclemont (Lepidoptera, Noctuidae) and impact of parasitism on food consumption of the host larvae

\author{
ANA C. YAMAMOTO \\ Augusta K. Doetzer \\ \& LUÍS A. FOERSTER
}

Ectoparasitóides do gênero Euplectrus Westwood caracterizam-se por sustar o processo de ecdise dos hospedeiros, levando-os à morte no mesmo ínstar em que foram parasitados (GERLING \& LIMON, 1976; PutTler, GordH \& Long, 1980; Coudron, Brandt \& Ragib, 1990). Resultados anteriores demonstram que apenas á injeção do fluido parálizante pela fêmea no momento do parasitismo é suficiente para suspender o desenvolvimento do hospedeiro, independentemente da presençąl dos parasitóides sobre o corpo do hospedeiro (PUTTLER, Gordı \& Lonvi, 1980). Esta característica indica que o parasitismo por Euplectrus causa um impacto significativo no desenvolvimento dos hospedeiros.

Deparameno de Zoologin, Sctor de Ciências Biologicas, UFPR - Caixal postal IS.020 $8 / .531 .990$, Curitiba. Paraná, Brasil. 
A lagalla do trigo, Pseudaletia sequax Franclemont, 1951 é parasitada no campo por cerca de oito espécies, incluindo dípteros da família Tachinidae e himenópteros das famílias Braconidae, Ichneumonidie e Eutophidae (GAssen, 1986; LINK \& CostA, 1988). Dentre os parasitóicles que atacam a lagarta do trigo no Brasil, a espécie maiss estudada é o braconídeo Glyptapanteles muesebecki (Blanchard) (Oliverra Filho \& Foerster, 1986; Doetzer \& Foerster, 1998; Fohrster, Avnnci \& Doetzer, 1999, prelo). Devido à inexistência de clados sobre a biologia de Euplectrus ronnai (Brèthes), e dada a grande incidência de lagartas de $P$. sequax parasitadas por esta espécie en cercais de inverno no sul do Paraná, determinou-seo efeito da temperatura no desenvolvimento de E. ronnat e o impacto do parasitismo no consumo alimentar de $P$. sequax.

\section{MATERIALE MÉTODOS}

Os experimentos foram realizados no Laboratório de Controle Integrado de Insetos (L. C. I. I.) do Departamento de Zoologia da UFPR. Exemplarcs de E. ronnai foram depositados no Museu de Entomologia "Jesus Santiago Moure" do Departamento de Zoologia da UFPR.

EFEITO DA TEMPERATURA NO DESENVOLVIMENTO - Lagartas de $4^{9}$ instar de $P$. sequix foram expostas a fêmeas de $E$. ronnai por 24 horas, a $21^{\circ} \pm 1{ }^{\circ} \mathrm{C}$, umidade relativa de $70 \pm 5 \%$ e fotofase de 12 horas. Após este período, as lagartas parasitadas foram individualizadas em potes de polietileno e transferidas para câmaras climatizadas a $15^{\circ}, 17^{\circ}, 21^{\circ}, 25^{\circ} \mathrm{e} 29^{\circ} \pm 1^{\circ} \mathrm{Ce}$ U. R. de $70 \pm 5 \%$. Utilizou-se 12 horas de fotofase para as temperaturas de $15^{\circ}, 17^{\circ} \mathrm{e} 21^{\circ} \mathrm{Ce} 14$ horas de fotolase para as temperaturas de $25^{\circ}$ e $29^{\circ} \mathrm{C}$. As lagartas for:um alimentadas com folhas de capim quicuio (Pennisetum clandestinum Hochstetter) e a duração dos estágios de ovo-larva e pupa, a lém da mortalidade, foram avaliadas em cada temperatura. A sobrevivência de E. ronnai em cada temperatura foi comparada pelo teste de $\chi^{2}(p \leq 0,05)$. Os limites térmicos de desenvolvimento inferior e as necessidades térmicas foram estimados pela equação de regresSĩo linear (HADDAD \& PARRA, 1984). 
I MPACIO DO PARASITISMO NO CONSUMO ALIMENTAR DO HOSPEDEIRO - Avaliou-se o consumo alimentar de lagartas de $P$. sequax parasitadals 110 quarto ínstar por $E$. ronnai e determinou-se o efeito do fluido paralizante da fêmea do parasitóide através da retirada dos ovos imedialamente após sua deposição sobre as lagartas. As lagartas foram individualizadas em potes de polietileno, acondicionadas em câmara climatizada a $21^{\circ} \pm 1^{\circ} \mathrm{C}$, com fotofase de 12 horas, e alimentadas com folhas de capim quicuio. O parasitismo foi realizado na mesma temperatura, exponclo-se fêmeas de E. ronnci a 40 lagartas de $4^{\circ}$ ínstar de P. sequax durante 24 horas, no mesmo dia em que loi observada a ecdise das lagartas. Após este período, os ovos do parasitóide foram rcmovidos de metade das lagartas, sob lupa e com o alxílio de um estilete, e iniciou-se a avaliação da quantidade diária de alimento consumido. Diariamente, foram pesadas folhas frescas ofertadas às lagartas e após 24 horas, o alimento não consumido foi levado à estufa entre $70^{\circ} \mathrm{C} 80^{\circ} \mathrm{C}$ por 48 horas e pesado em seguida. O peso seco do alimento ofertado foi estimado através da multiplicação do peso fresco das folhas fornecidas pela percentagem média do peso seco de umal folha, obtida pela média do peso seco de 20 folhas. A quantidade diária de alimento consumido pelas lagartas foi calculada subtraindo-se o peso seco do alimento não consumido do peso seco do alimento ofcrtado. Este procedimento foi seguido até a pupação dos parasitóides ou até a morte das lagartas, naquelas em que foram removidos os ovos dc E. ronnai. Os resultados foram comparados com o consumo de lagartas não parasitadas obtido por DoETzER \& FoERSTER (1998), utilizando a mesma metodologia para a determinação do consumo alimentar:

\section{RESULTADOS E DISCUSSÃO}

EliEITO DA TEMPERATURA NO DESENVOLVIMENTO - O parasitóide completou o desenvolvimento em todas as temperaturas estudadals, porém a sobrevivência de $E$. ronnai foi significativamente influenciada pela temperatura (Tabela 1). Registrou-se uma relação diretamente proporcional entre a temperatura $\mathrm{e}$ a porcentagem de sobrevivência até $\mathrm{o}$ estágio adulto, entre $15^{\circ}$ e $25^{\circ} \mathrm{C}$; a menor sobrovivência $(52 \%)$ for obticla a $15^{\circ} \mathrm{C}$, atingindo $87 \%$ a $25^{\circ} \mathrm{C}$. A $29^{\circ} \mathrm{C}$, a sobrevivência 
voltou a diminuir, indicando que temperaturas acima de $25^{\circ} \mathrm{C}$ s s̃o prejudiciais a E. ronnai. Estatisticamente, não houve diferença significativa na sobrevivência entre $21^{\circ}$ e $29^{\circ} \mathrm{C}$, enquanto que, a $15^{\circ}$ e 17 ${ }^{\circ} \mathrm{C}$, a porcentagem de sobrevivência foi significativamente menor que a $25^{\circ} \mathrm{C}$. Em todas as temperaturas, exceto a $25^{\circ} \mathrm{C}$, a maior porcentagem de mortalidade ocorreu no estágio larval, a qual variou entre $4,3 \%$ a $25^{\circ} \mathrm{Co} 31,0 \%$ a $15^{\circ} \mathrm{C}$ (Tabela 1). Lagartas de $P$. sequax parasitadas pelo braconídeo G. muesebecki também sobreviveram entre $14^{\circ}$ e 29 ${ }^{\circ} \mathrm{C}$; no entanto, a $30^{\circ} \mathrm{C}$, embora ocorresse parasitismo por $G$. muesebecki, as lagartas morreram antes que o parasitóide empupasse (Foerster, Avanci \& Doetzer, 1999, prelo).

A duração da tase de ovo-larva variou entre 6,0 dias a $29^{\circ} \mathrm{C} \mathrm{e}$ 28,2 dias a $15^{\circ} \mathrm{Ce}$ a duração do ciclo total de E. ronnai variou de $11,9\left(29^{\circ} \mathrm{C}\right)$ a 62,7 dias $\left(15^{\circ} \mathrm{C}\right)$. Em todas as temperaturas, a duração dos estágios de ovo-larva foi semelhante à duração do estágio pupal (Tabela 2), ao contrário de G. muesebecki, onde o estágio de ovo-larva foi cercal de duas vezes mais longo que o período pupal (OLIVEIRA FIL 10 \& Foerster, 1986; DoETZER \& FoERSTER 1998; Foerster, Avanci \& Doetzer, 1999, prelo). Outras espécies de Euplectrus também apresentam a mesma proporcionalidade na duração do estágio ovo-larval em comparação à fase pupal (GERLıNG \& Limon 1976 , Puttler, Gordh \& Long, 1980, Menezes Jínior, Migurl \& Berbei, 1994; Coudron, Brandt \& RaQib, 1997). A maior velocidade de desenvolvimento dos dois primeiros estágios de E. ronnai em compáração a $G$. mueschecki significa maior eficiência desse parasitóide com relação alos danos provocados pelo hospedeiro, visto que a lagarta morre ao final do estágio larval do parasitóide. A $21^{\circ} \mathrm{C}$, a morte de $P$. sequax por E. romnai ocorre em média 12 dias após o parasitismo, cerca da metade do tempo despendido por $G$. muesebecki para causar a morte do hospedeiro, o qual foi de 23,7 dias, na mesma temperatura (Doetzer \& Foerster, 1998).

Plittler, Gurdh \& Long (1980) verificaram para o ciclo total de $E$. puttleri uma variação de 10,0 a 44,2 dias nas temperaturas de $30^{\circ} \mathrm{e}$ $15^{\circ} \mathrm{C}$, respectivamente. Comparativamente a $E$. ronnai, o desenvolvimento de E. puttlerié mais acelerado, reflexo da menor duração do estágio larval do hospedeiro; enquanto Anticarsia gemmatalis. Hübner 
completal o estágio larval em 13 dias a $27^{\circ} \mathrm{C}$ (MAGrint et al., 1996), P. socquax completa esta fase em 23,5 dias a $26^{\circ} \mathrm{C}$ (Foerster, 1996).

A temperatura base ( $\mathrm{Tb}$ ) foi de $11,4^{\circ} \mathrm{C}$ para oestágio de ovo-larva e de $12,3^{\circ} \mathrm{C}$ para o estágio de pupa (Tabela 3 , Fig. 1), demonstrando que a fasc de pupá mais suscetíve] a baixas temperaturas, como também observado para G. muesebecki (Foerster, Avavel \& DofitZer, 1999, prelo). O limite térmico inferior do ciclo total de E. ronnai foi de $11,9{ }^{\circ} \mathrm{C}$ (Tabela 3; Fig. 1), superior alo de seu hospedeiro P. sequa $x$, delerminalo em $9,3^{\circ} \mathrm{C}$ por Foerster (1996). A constante térmica (K) foi de 109,5 GD e 102,4 GD para os estágios de ovo-larva e pupa, respectivamente, enquanto que o ciclo cvolutivo requercu um total de 211,4 GD para ser completado (Tabela 3). Para G. muesehecki, Forisstir, A vanci \& Doetzer (1999, prelo) constataram o limiar térmico de desenvolvimento inferior e a constante térmica para o ciclo total, sendo de $9,6^{\circ} \mathrm{Ce} 397,4 \mathrm{GD}$, respectivamente. Verifica-se que E. romai necessita de 186 GD a menos do que G. muesebecki, para completar seu ciclo de desenvolvimento. Portanto, do ponto de vista de eficiência, $E$. ronnai ocasiona a morte da lagarta antes de $G$. muesebecki, diminuindo os danos causados por $P$. sequax. Comparando-se o limiar térmico dos dois parasitoóides, conclui-se que $G$. muesele cki é ativo em temperaturas mais baixas em relação a E. ronnai.

IMIACTO DO PARASITISMO NO CONSUMO ALIMENTAR DO HOSPEDEIRO - Ao contrário de G. muesebecki, o parasitismo por E. ronnai inibe a ecdise do hospedeiro, e as lagattas parasitadas por essa espécie morrem no mesmo ínstar em que foram parasitadas. Com os parasitóides mantidos sobre o hospedeiro, o consumo médio ( \pm E. P.) de lagartas parasitadas no $4^{\underline{1}}$ instar foi de $37,7 \pm 2,67 \mathrm{mg}$ de peso seco do alimento, valor semelhante ao encontrado para lagartas das quais foram removidos os ovos do parasitóide logo após o ataque por E. ronnai $(40,7 \pm 3,54 \mathrm{mg})$. DOETzer \& Foerster (1998) registralam para lagartas não parasitadas de P. sequax um consumo médio de 791,9 [4,05 $\mathrm{mg}$ de peso seco de alimento durante o estágio larval. Desse total, mais de $90 \%$ foi consumido a partir do 4 "instar, estádio onde lagartas de $P$. sequax tornam-se prejudiciais economicamente. Estes resultados demonstram que E. ronnai reduz em cerca de $95 \%$ o consumo alimentar de lagartas de $P$. sequax, índice acentuadamente maior 
Tebela 1. Mordidiade f\%) nos estígios de ovo, larva e pupa de Euplectras mmai sobre

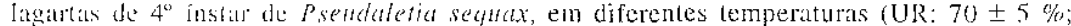
Fototuse: 12 horak paral $15 ", 17 " 021^{\circ} \mathrm{C}$ \& 14 horas para $25^{\circ} \circ 20^{\circ} \mathrm{C}$ ).

\begin{tabular}{cccccc}
\hline Temperatura $\left({ }^{\circ} \mathrm{C}\right)$ & & \multicolumn{4}{c}{ Mortalidade $(\%)$} \\
\cline { 3 - 6 } & $\mathrm{N}$ & Ovo & Larva & Pupa & Total \\
\hline 15 & 17 & 10,0 & 31,0 & 7,0 & 48,0 \\
17 & 32 & 15,2 & 22,5 & 0 & 38,0 \\
21 & 37 & 2,1 & 14,7 & 4,2 & 21,0 \\
25 & 40 & 6,5 & 4,3 & 2,2 & 13,0 \\
29 & 45 & 3,0 & 25,3 & 1,7 & 30,0 \\
\hline
\end{tabular}

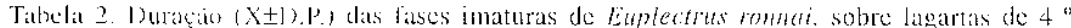

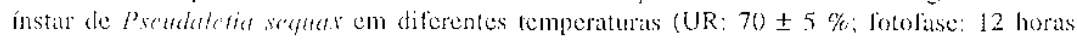

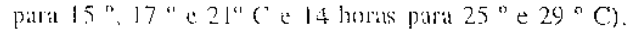

\begin{tabular}{|c|c|c|c|c|}
\hline \multirow{3}{*}{ Temperatura $\left({ }^{\circ} \mathrm{C}\right)$} & \multirow{3}{*}{$\begin{array}{l}N \\
17\end{array}$} & \multicolumn{3}{|c|}{ Duração das fases (dias) ${ }^{1}$} \\
\hline & & \multirow{2}{*}{$\frac{\text { Ovo-larva }}{28,2 \pm 1,1 \text { a }}$} & \multirow{2}{*}{$\begin{array}{c}\text { Pupa } \\
34,5 \pm 2,0 a\end{array}$} & \multirow{2}{*}{$\frac{\text { Total }}{62,7 \pm 2,7 a}$} \\
\hline & & & & \\
\hline 17 & 32 & $18,8=1,0 b$ & $21,2=0,8 b$ & $40,0 \pm 1,3 b$ \\
\hline 21 & 37 & $12,0 \pm 0,6 \mathrm{c}$ & $12,5 \pm 0,6 \mathrm{c}$ & $24,5 \pm 0,7 \mathrm{c}$ \\
\hline 25 & 40 & $8,7 \pm 0,5 d$ & $8,4 \pm 0,5 d$ & $17,1 \pm 0,4 d$ \\
\hline 29 & 45 & $6,0 \pm 0,6 \mathrm{e}$ & $6,0 \pm 0,6 \mathrm{e}$ & $11,9 \pm 0,4 \mathrm{e}$ \\
\hline
\end{tabular}

Tabela 3. Tenperatura base (Th), constante tótuica (K), oguaçäo da velocidade do deservol-

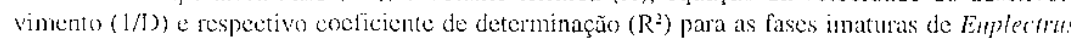

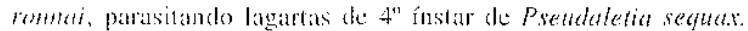

\begin{tabular}{lcccc}
\hline Fases & Tb $\left({ }^{\circ} \mathrm{C}\right)$ & $\mathrm{K}(\mathrm{GD})^{\prime}$ & $\mathrm{Equação}^{2}$ & $\mathrm{R}^{2}(\%)$ \\
\hline Ovo-larva & 11,4 & 109,5 & $1 / \mathrm{D}=-0,104378+0,009135 \mathrm{~T}$ & 98,6 \\
Pupa & 12,3 & 102,4 & $1 / \mathrm{D}=-0,120218+0,009762 \mathrm{~T}$ & 99,4 \\
Ciclo & 11,9 & 211,4 & $1 / \mathrm{D}=-0,056354+0,004730 \mathrm{~T}$ & 99,1 \\
\hline
\end{tabular}

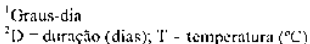


Arfu Biol: Par, Curitiba, 27 (1,2,3,4): 85-95. 1998
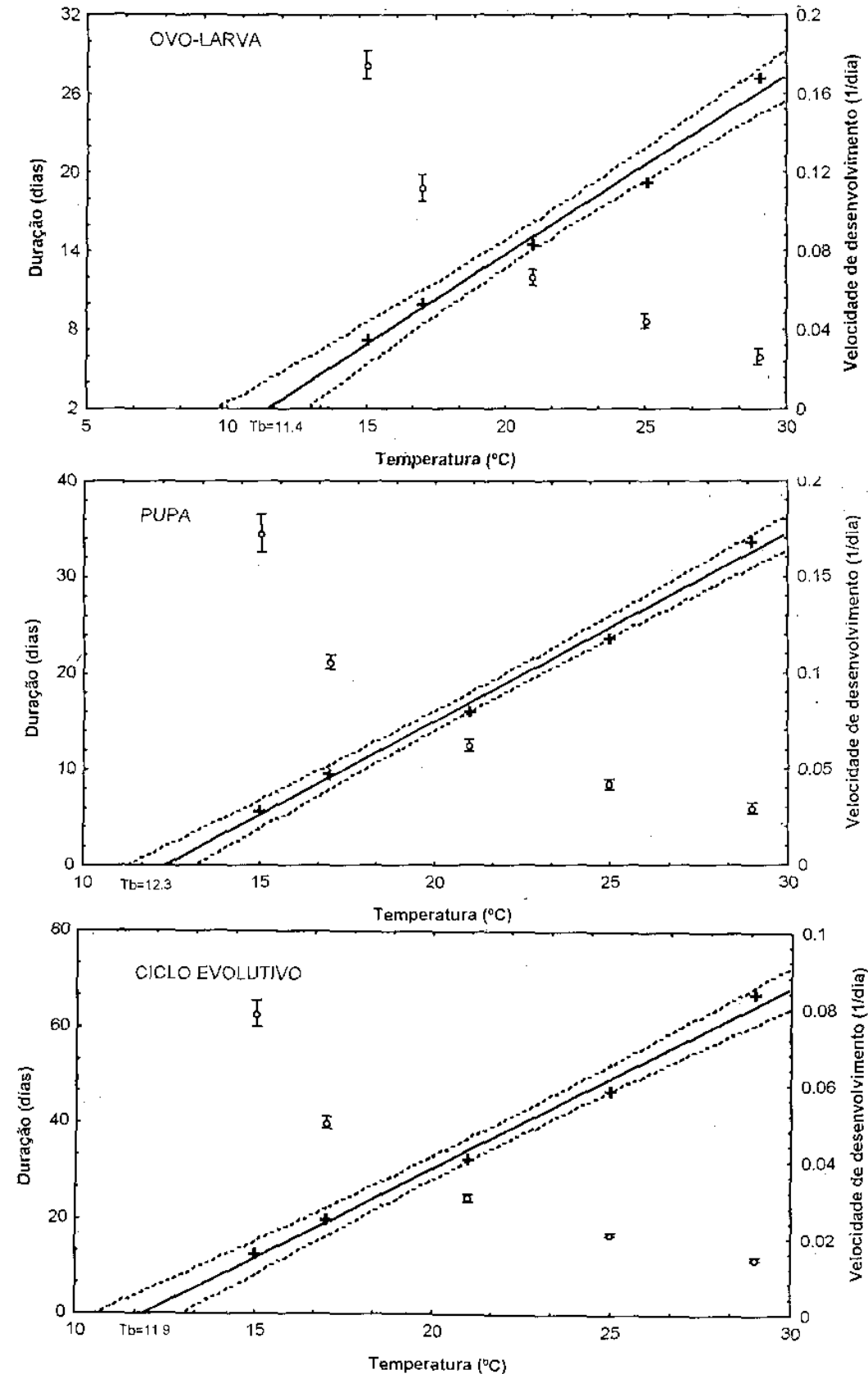

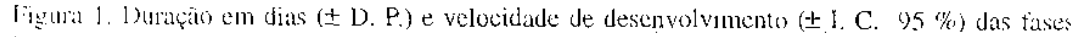

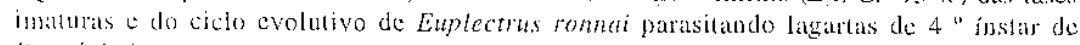
prestateria realus. 
que o provocaldo por $G$. muesebecki, de $41 \%$ (DOETzER \& Foerster 1998). Para outras espécies de Euplectrus, Parkman \& Shepard (1981) verificaram que o parasitismo por E. plathypenae Howard reduz em cercal de $86 \%$ o consumo foliar de lagartas de Spodoptera omithogalli (Guené).

Os resultidos demonstram que apenas a injeção do fluido paralizante é suliciente para inibir a ecdise e reduzir o consumo de alimento aos mesmos níveis registrados em lagat tas que hospedam parasitóides. Apesar do baixo consumo, lagartas cujos ovos dos parasitóides foram removidos sobreviveram significativamente mais ( 30 dias) do que lagartas em quc os parasitóides foram mantidos sobre o tegumento (12 dias). A supressão da cedise c a ação inibitória do fluido paralizante sobre o desenvolvimento também foi constatada em $E$. puttleri (Puttllik, Gorin \& Long, 1980).

As característicals de E. ronnai permitem concluir que este parasitóide causa um impacto significativo no consumo de $P$. sequax, constituincto-se em um eficicnte agente de controle da lagarta do trigo.

\section{RESUMO}

Estudou-se em Jaboratório o efeito de cinco temperaturas constantes $\left(15^{\circ}, 17^{\circ}, 21^{\circ}, 25^{\circ} \mathrm{e} 29^{\circ} \mathrm{C}\right)$ na duração das fases imaturas de Euplectrus ronnat (Brèthes) sobre lagartas de Pseudaletia sequax Franclemont. $\mathrm{O}$ parasitóide completou o desenvolvimento em todas as temperaturas. A menor mortalidalde $\left(1.3 \%\right.$ ) ocorreu a $25^{\circ} \mathrm{C}$ e a maior $(48 \%)$ a $15^{\circ} \mathrm{C}$. Obleve-se uma relação linear entre o tempo de desenvolvimento de $E$. ronnai c a temperatura na faixa entre $15^{\circ} \mathrm{C} 29^{\circ} \mathrm{C}$. A temperatura base $(\mathrm{Tb})$ e a constante térmica $(\mathrm{K})$ foram estimadas através da equação de regressão lineal; a $\mathrm{Tb}$ foi de $11,4^{\circ} \mathrm{C}$ para a fase ovo-larval e $12,3^{\circ} \mathrm{C}$ para a faste pupal. A constante térmica para a fase ovo-larval e pupal foi de 109,5 c 102,4 graus-dia (GD), respectivamente, e 211,4 GD durante todo o período de desenvol vimento. A temperatura mais adequada para o desenvolvimento de E. ronnai, em termos de sobrevivência e de velocidade de desenvolvimento foi $25^{\circ} \mathrm{C}$. O consumo alimentar de lagartas parasitadals foj avaliado em laboratório, utilizando-se folhas capim quicuio (Pennisenm clandestinum Hochstetter) como alimento. Lagartas parasitadas consumiram cerca de $95 \%$ menos alimento que lagartas não parasitadas, mesmo quando os ovos do parasitóide foram retirados logo 
após a oviposição, demonstrando que o desenvolvimento do hospedeiro é drasticamente reduzido pela injeção do fluido paralizante no momento do parasitismo.

Pá.avras chave: Insecta, ectoparasitóide, lagarta do trigo, controle biolów gico.

\section{SUMMARY}

The effect of five constant temperatures $\left(15^{\circ}, 17^{\circ}, 21^{\circ}, 25^{\circ}\right.$ and $29^{\circ}$ C) on the development of Euplectrus ronnai (Brèthes) parasiting larvae of Pseudaletia sequax Franclemont was carried out in the laboratory. The development of the parasitoid was completed in all temperatures. Lowest mortality ( $13 \%$ ) was recorded at $25^{\circ} \mathrm{C}$ and highest $(48 \%)$ at 15 ${ }^{\circ} \mathrm{C}$. A linear relationship was observed in the development of $E$. ronnai in the range between $15^{\circ}$ and $29^{\circ} \mathrm{C}$. The lower threshold temperature (Tb) and the thermal constant (K) were evaluated using the linear regression equation. The lower threshold temperature was $11.4^{\circ} \mathrm{C}$ for the egg+larval stages and $12.3^{\circ} \mathrm{C}$ for the pupal stage. The thermal constant for the egglarval and pupal stages was 109.5 e 102.4 day-degrees (DD), respectively, and $211.4 \mathrm{DD}$ from oviposition to adult emergence. The most adequate temperature for laboratory rearing of $E$. ronnai, was $25^{\circ} \mathrm{C}$. Food consumption for parasitized larvae was evaluated in laboratory, using kikuyo grass (Pennisetum clandestinum Hochstetter) as larval food. Parasitized larvie consumed ca. $95 \%$ less foliage than unparasitized ones. Removal of parasitoid eggs from the host also reduced food consumption of the host in similar proportion. The results show that host growth is impaired by the injection of fluid during oviposition.

KEY WoRDS: Insecta, ectoparasitoid, armyworm, biological control.

\section{RÉSUMÉ}

La vitesse de développement des stades immatures de l'ectoparasitö̈de grégaire Euplectrus ronnai (B rèthes) en parasitant larves de Pseudaletia sequatix Franclemont a été estimée sous cinq températures constantes $\left(15^{\circ}, 17^{\circ}, 21^{\circ}, 25^{\circ}\right.$ et $\left.29^{\circ} \mathrm{C}\right) . \AA$ A toutes les températures, le parasitoïde a terminé son développement. La mortalité a été la plus faible $(13 \%)$ à 25 ${ }^{\circ} \mathrm{C}$ et la plus importante $(48 \%)$ à $15^{\circ} \mathrm{C}$. On a observé une relation lineaire entre le temps de développement de E. ronnai et la température. Le stade oeuf-larvaire a eu besoin de 109,5 degrés-jours au-dessus d'une température-seuil de $11,4^{\circ} \mathrm{C}$ pour terminer le développement, tandis que 
les nymphes ont cu besoin de 102,4 degrés-jour all-dessus de $12,3^{\circ} \mathrm{C}$. La tempéralture la plus: convenable pour l'élevage de ce parasitö̈de est $25^{\circ}$ C. On a étudié en laboratoire l'influence du parasitisme sur la consommation de nourriture de l'hôte. Les chenilles parasitées ont consomme a peu pres $95 \%$ moins de nourriture que les chenilles non parusitées.s même quand les oeufs du parasitoïde sont rétirés immédiatement après l'oviposition, en montrant que le développement de l'hôte est réduit pau l'injection du fluide paralysant au moment du parasitisme.

Mots ruks: Insecta, cetoparasitö̈de, chenille, lutte biologique.

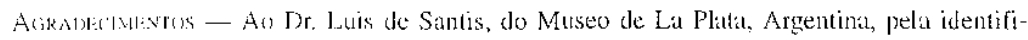

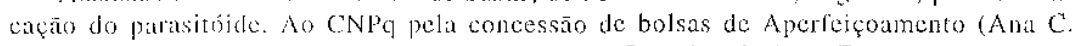

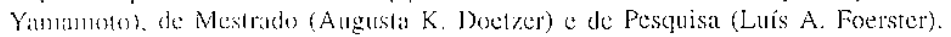

\section{BIBLIOGRAFIA}

Courrom, T.A., S.L. Branm \& A. Ragib, 1997. Comparison of the response of Heliothis virescens to parasitism by Euplectrus comstockit and Euplectris plathypenae. Comp. Biochen. Physiol. $1 / 6 B: 197-202$.

Doitrak, A. K. \& L.A. Fofrster. 1998. Efeito do parasitismo por Glyptapanteles muesebecki (Blanchard) no consumo e utilização do alimento por $P$ seudaletia sequax Franclemont. An. Soc. Entomol. Brasil, 27: 255-264.

Fofrster, L. A. 1996. Efeito da temperatura no desenvolvimento das fuses imaturas de Pscudaletia sequax Franclemont (Lepidoptera: Noctuidite). An. Soc. Entomol. Brasil 25: 27-32.

Fotrśster. L. A., M.R.F. Avanci \& A.K. DoetzLer. 1999. Effect of temperature on the development and progeny production of Glypapanteles muesebecki (Blanchard) (Hymenoptera: Braconidac) parasitizing larvae of Pseudaletic sequax Franclemont (Lepidoptera: Noctuidae). An, soc. Entomol. Brasil 28: 243-249.

Gassi:n, D. N. 1986. Insetos associados à cultura do trigo no Brasil. Passo Fundo, EMBRAPA-CNPT, 39p. (EMBRAPA-CNPT, Circular Técnica, 2).

GLikJ.ING, D. \& S. LIMON, 1976. A biological teview of the genus Euplectrus: (Hymenoptera : Eulophidae) with special emphasis on E. laphygmae as a parasite of Spodoptera littoralis (Lepidoptera : Noctuidae). Entomophatisa 21: 179-187. 
Halman, M. L. \& J. R. P. PARkA. 1984. Métodos para estimar os limites fómicos e a fatra ótima de desenvolvimento das diferentes ferses do ciclo de desenvolvimento de insetos. Piracicaba, Fundaçío de Estudos Agrários "Luiz de Queiroz", 12 p. (Sérje Agriculuma c Deschvolvimento).

Hns., M. (i. 1986. Fflects of Cote'sia rufichus (Braconidae: Hymenoptera) parasitism and rearing density on Myhmme separta (Noctuidate: Lepidopteral) food consumption, and implications for biological control. N. Z. J. Agric: Res. 29: 281-288.

Link. D. \& E. C. Costa. 1988. Aspectos biológicos das lagartas do trigo. Rev. Cent. Ciênc. Rurctis 18:99-105.

Magrini, E. A., S. S. Neto, J. R. P. Parka, P. S. M. Botelho \& M. L. HanbaD. 1996. Biologia e exigências térmicas de Anticarsia gemmatalis Hübner (Lepidoptera: Noctuidate) em laboratório. An. Sox: Entomol. Brasil 25: 513-519.

Mentaks Jinior, A. O.; M. Migulaz \& B. M. Birrat. 1994. Biologia de Fupectrus puttleri Gordh, 1980 (Hymenoptera, Eulophidae) sobre lagartas do curuquere do algodoeiro, Alabana argilaceae Hübner, 1818 (Lepidoptera, Noctuidae). Seniná Ciên. Agr. 15: 23-28.

Mrssinger, P.S. 1959. Bioclimatic studies with insects. Annu. Rev. Fintomol. 4: 183-206.

Morales, J. \& A. A. Hower. 1981. Thermal recuirements for development of the parasite Microctonus athiopoides. Environ. Entomol. 10: $279-284$.

Olvinka Fling, I. \& L, A. Fonkster. 1986, Ciclo evolutivo e preferencial para oviposicăo de Apanteles musebecki Blanchard, 1947 (Hymenoptera, Braconidae), parasitóide de PSeudaletia sequax Fanclemont. 1951 (Lepidoptera, Noctuidae). An. Soc. Entomol. Brasil $15: 371-376$.

PNRKMAN, P. \& M. SHEPARL. 1981, Foliage consumption by yellowstriped armyworm larvale after parasitization by Euplectras plathyperae. Florida Entomologist 64 (1): 192-194.

PuTthar, B. C., C. Goridi \& S. H. Long. 1980. Bionomics of Euplectras futher Gordh, new species, an introciuced parasite of the velvetbean caterpillar, Anticarsia gemmatalis from South America. Ann. Entomol. Soc: Am. $73: 28-35$.

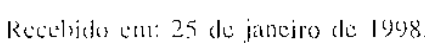

